Journal of Mathematics and Statistics 5 (2):112-117, 2009

ISSN 1549-3644

(C) 2009 Science Publications

\title{
Stochastic Behavior of Redundant Complex System with Two Types of Failure
}

\author{
${ }^{1}$ G.S. Mokaddis, ${ }^{1}$ M. Salah EL-Sherbeny and ${ }^{2}$ Entesar Al-Esayeh \\ ${ }^{1}$ Department of Mathematics, Faculty of Science, Ain Shams University, Cairo, Egypt \\ ${ }^{2}$ Department of Statistics, Faculty of Science, +7 th April University, Zawia, Libya
}

\begin{abstract}
Problem statement: This study presented the reliability and Mean Time To System Failure (MTTF) analysis of a two-state complex with repairable system, consisting of two sub-systems A and $\mathrm{B}$ arranged in series, incorporating the concept of hardware and human failures. The failure times of operating units and repair time of failed units were exponential distributed. Approach: Markovrenewal processes and properties of Laplace transforms had been used to measure the system effectiveness Results: Laplace transforms of the various state probabilities had been derived and then reliability of the complex system, at any time $t$, had been computed by inversion process. MTTF had also been evaluated; availability and steady-state availability for system was derived. Certain important result had been evaluated as special cases. Also, few graphical illustrations were also given at the end to high-light the important results. Conclusion: The additional repair led to improve the values of the MTTF and the reliability at any time $t$.
\end{abstract}

Key words: Reliability to system R(t), Availability to system A(t) and mean-time to failure

\section{INTRODUCTION}

Earlier researchers ${ }^{[1,2]}$ have studied the reliability and MTTF for various complex equipments, keeping in view the concept of human and hardware failure ${ }^{[3]}$, have studied the Reliability and MTTF analysis of nonrepairable parallel redundant complex system under hardware and human failures ${ }^{[3]}$, have studied the Human error and partial hardware failure modeling of parallel and standby redundant system and ${ }^{[4]}$ have studied the stochastic analysis of a compound redundant system involving human failure As a matter of fact human failure is defined as a failure to perform a prescribed task which could result in damage to the equipment and property. There exist a number of causes for human error; e.g., lack of good job environments, poor training or skill of the operating personnel and so on. On the other hand, hardware failure occurs due to flaws in design, poor quality control, poor maintenance. This type of study can be found in reference. In this study; the researchers have considered a repairable complex system consisting of two subsystems A and B (Fig. 1). The subsystem A has a two-unit active parallel system whereas the subsystem B has one unit alone. The two subsystems are arranged in series. Both the units of subsystem A suffer two types of failure viz; hardware and human whereas subsystem B suffers only one type of failure. With the aid of Laplace transforms of the various state probabilities have been derived and then reliability is obtained by inversion process. Moreover, an important parameter of reliability, i.e., MTTF (mean time to failure), system availability and steady-state availability are derived. The failure times of operating units and repair time of failed units are exponential distributed. The effects of additional repair in this system performance are shown in tables and graphically.

\section{MATERIALS AND METHODS}

Assumptions: In this system the following assumptions and notations are used to analyze the system:

- Initially, the system is in good state

- The system has two states, viz; good and failed

- A failed unit can be repaired

- Hardware failures and human failures for all the units are also constant

- Failures are statistically independent

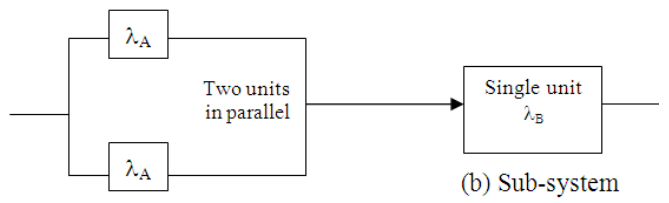

(a) Sub-system

Fig. 1: Connect between two sub-system

Corresponding Author: G.S. Mokaddis, Department of Mathematics, Faculty of Science, Ain Shams University, Cairo, Egypt 


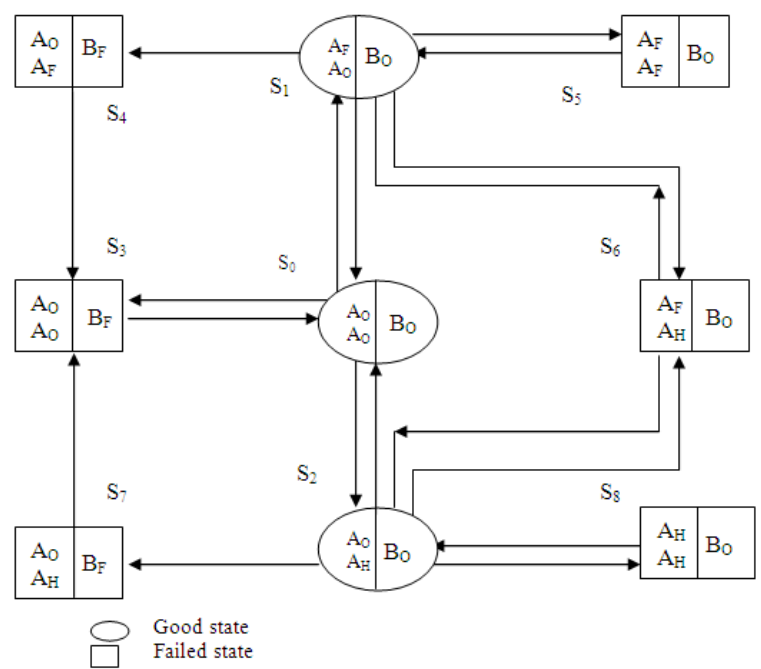

Fig. 2: State transition diagram

- Two units connected in parallel redundancy suffer two types of failures; namely constant hardware failure and constant human failure

- In the complex system, only one change can take place in the state of the system at any time

Symbol for the modes: O operable mode, F hardware failures mode and $\mathrm{H}$ human failure mode.

Analysis of the system: To analyze the behavior of the system, we note that at any time $t$, the system will be found in any one of the above in (Fig. 2) mutually exclusive and exhaustive states:

$\mathrm{S}_{0} \quad$ Two-unit of sub-system a are operating and the other sub-system B is operating

$\mathrm{S}_{1} \quad$ One-unit of sub-system $\mathrm{A}$ is failed with hardware failure, second unit of sub-system A is operating and sub-system B is operating

$\mathrm{S}_{2}$ One-unit of sub-system $\mathrm{A}$ is failed with human failure, second A unit operating and sub-system B is operating

$\mathrm{S}_{3}$ Two-unit of sub-system A are operating and the other sub-system B is failed

$\mathrm{S}_{4} \quad$ One-unit of sub-system $\mathrm{A}$ is failed with hardware failure, second unit of sub-system A is operating and sub-system $B$ is failed

$\mathrm{S}_{5}$ Two-unit of sub-system $\mathrm{A}$ are failed with hardware failure and sub-system B is operating

$\mathrm{S}_{6} \quad$ One-unit of sub-system A is failed with hardware failure, second unit of sub-system A is failed wit human failure and sub-system B is operating
$\mathrm{S}_{7}$ One-unit of sub-system $\mathrm{A}$ is failed with human failure, second unit of sub-system $\mathrm{A}$ is operating and sub-system $B$ is failed

$\mathrm{S}_{8} \quad$ Two-unit of sub-system A are failed with human failure and the other sub-system B is operating

$\mathrm{p}_{\mathrm{i}}(\mathrm{t})$ Probability that the system is in state $\mathrm{s}_{\mathrm{i}}$ at any time $\mathrm{t}$, for $\mathrm{i}=0,1,2, \ldots, 8$

s Laplace-transform variable

$\mathrm{F}(\mathrm{s})$ Laplace-transform of $\mathrm{F}(\mathrm{t})$

$\lambda_{A}$ The constant hardware failure rate of a unit of sub-system A

$\lambda_{h}$ The constant human failure rate of a unit of subsystem A

$\lambda_{B}$ The constant hardware failure rate of sub-system B

$\mu_{\mathrm{A}}$ The constant repair rate from hardware failure of a unit for the sub-system A

$\mu_{\mathrm{h}}$ The constant repair rate from human failure of a unit for the sub-system A

$\mu_{\mathrm{B}} \quad$ The constant repair rate of sub-system B

\section{RESULTS AND DISCUSSION}

System reliability: The system reliability $R(t)$ is the probability of failure-free operation of the system in $(0$, t]. To derive an expression for the reliability of the system, we restrict the transitions of the Markov process to the up states, viz. $S_{0}, S_{1}, S_{2}, S_{3}, S_{4}$ and $S_{5}$. Using the Fig. 2, we derive the following differential equations:

$$
\begin{aligned}
& \frac{\mathrm{dp}_{0}(\mathrm{t})}{\mathrm{dt}}+\left(2 \lambda_{\mathrm{A}}+2 \lambda_{\mathrm{h}}+\lambda_{\mathrm{B}}\right) \mathrm{p}_{0}(\mathrm{t})=\mu_{\mathrm{A}} \mathrm{p}_{1}(\mathrm{t})+\mu_{\mathrm{h}} \mathrm{p}_{2}(\mathrm{t}), \\
& \frac{\mathrm{dp}_{1}(\mathrm{t})}{\mathrm{dt}}+\left(\lambda_{\mathrm{A}}+\lambda_{\mathrm{h}}+\lambda_{\mathrm{B}}+\mu_{\mathrm{A}}\right) \mathrm{p}_{1}(\mathrm{t})=2 \lambda_{\mathrm{A}} \mathrm{p}_{0}(\mathrm{t}) \\
& \frac{\mathrm{dp}_{2}(\mathrm{t})}{\mathrm{dt}}+\left(\lambda_{\mathrm{A}}+\lambda_{\mathrm{h}}+\lambda_{\mathrm{B}}+\mu_{\mathrm{h}}\right) \mathrm{p}_{2}(\mathrm{t})=2 \lambda_{\mathrm{h}} \mathrm{p}_{0}(\mathrm{t})
\end{aligned}
$$

$$
\text { Let }_{\mathrm{P}_{\mathrm{i}}}(\mathrm{s}) \text { be the Laplace transform of }
$$
$p_{i}(t), i=0,1,2$. Taking Laplace transform on both the sides of the differential Eq. 1 and using the initial conditions $P_{0}(0)=1, \quad P_{i}(0)=0$, where $i=1,2$, solving for $P_{i}(s) ; i=0,1,2$, we get:

$$
\begin{aligned}
& \mathrm{p}_{0}(\mathrm{~s})=\frac{\left(\mathrm{s}+\mathrm{x}_{2}\right)\left(\mathrm{s}+\mathrm{x}_{3}\right)}{\prod_{\mathrm{r}=1}^{3}\left(\mathrm{~s}-\mathrm{s}_{\mathrm{r}}\right)}, \mathrm{p}_{1}(\mathrm{~s})=\frac{2 \lambda_{\mathrm{A}}\left(\mathrm{s}+\mathrm{x}_{3}\right)}{\prod_{\mathrm{r}=1}^{3}\left(\mathrm{~s}-\mathrm{s}_{\mathrm{r}}\right)} \\
& \mathrm{p}_{2}(\mathrm{~s})=\frac{2 \lambda_{\mathrm{h}}\left(\mathrm{s}+\mathrm{x}_{2}\right)}{\prod_{\mathrm{r}=1}^{3}\left(\mathrm{~s}-\mathrm{s}_{\mathrm{r}}\right)}
\end{aligned}
$$


Taking inverse L.T. of Eq. 2, we get the $\mathrm{p}_{\mathrm{i}}(\mathrm{t}) \cdot \mathrm{i}=0,1,2$ :

$$
\begin{aligned}
& \mathrm{p}_{0}(\mathrm{t})=\sum_{\mathrm{i}=1}^{3} \frac{\left(\mathrm{s}_{\mathrm{i}}+\mathrm{x}_{2}\right)\left(\mathrm{s}_{\mathrm{i}}+\mathrm{x}_{3}\right)}{\prod_{\mathrm{r}=1, \mathrm{r} \neq \mathrm{i}}^{3}\left(\mathrm{~s}_{\mathrm{i}}-\mathrm{s}_{\mathrm{r}}\right)} \mathrm{e}^{\mathrm{s}_{\mathrm{i}} \mathrm{t}}, \\
& \mathrm{p}_{1}(\mathrm{t})=\sum_{\mathrm{i}=1}^{3} \frac{2 \lambda_{\mathrm{A}}\left(\mathrm{s}_{\mathrm{i}}+\mathrm{x}_{3}\right)}{\prod_{\mathrm{r}=1, \mathrm{r} \neq \mathrm{i}}^{3}\left(\mathrm{~s}_{\mathrm{i}}-\mathrm{s}_{\mathrm{r}}\right)} \mathrm{e}^{\mathrm{s}_{\mathrm{i}} \mathrm{t}}, \\
& \mathrm{p}_{2}(\mathrm{t})=\sum_{\mathrm{i}=1}^{3} \frac{2 \lambda_{\mathrm{h}}\left(\mathrm{s}_{\mathrm{i}}+\mathrm{x}_{2}\right)}{\prod_{\mathrm{r}=1, \mathrm{r} \neq \mathrm{i}}^{3}\left(\mathrm{~s}_{\mathrm{i}}-\mathrm{s}_{\mathrm{r}}\right)} \mathrm{e}^{\mathrm{s}_{\mathrm{i}} \mathrm{t}}
\end{aligned}
$$

Then the system reliability is given by:

$$
\begin{aligned}
R(t) & =P_{0}(t)+P_{1}(t)+P_{2}(t) \\
& =\sum_{i=1}^{3} \frac{\left(s_{i}+x_{2}\right)\left(s_{i}+x_{3}\right)+2 \lambda_{A}\left(s_{i}+x_{3}\right)+2 \lambda_{h}\left(s_{i}+x_{2}\right)}{\prod_{r=1, r \neq i}^{3}\left(s_{i}-s_{r}\right)} e^{s_{1} t}
\end{aligned}
$$

where, $\mathrm{s}_{1}, \mathrm{~s}_{2}$ and $\mathrm{s}_{3}$ are roots of the following equation:

$$
\begin{aligned}
& \mathrm{s}^{3}+\mathrm{s}^{2}\left(4 \lambda_{\mathrm{A}}+4 \lambda_{\mathrm{h}}+3 \lambda_{\mathrm{B}}+\mu_{\mathrm{A}}+\mu_{\mathrm{h}}\right)+\mathrm{s} \\
& {\left[5 \lambda_{\mathrm{A}}^{2}+3 \lambda_{\mathrm{B}}^{2}+\lambda_{\mathrm{h}}\left(5 \lambda_{\mathrm{h}}+3 \mu_{\mathrm{A}}\right)+\mu_{\mathrm{h}}\left(\lambda_{\mathrm{h}}+\mu_{\mathrm{A}}\right)\right.} \\
& +2 \lambda_{\mathrm{B}}\left(4 \lambda_{\mathrm{h}}+\mu_{\mathrm{A}}+\mu_{\mathrm{h}}\right)+\lambda_{\mathrm{A}}\left(8 \lambda_{\mathrm{B}}+10 \lambda_{\mathrm{h}}+\mu_{\mathrm{A}}+3 \mu_{\mathrm{h}}\right) \\
& {\left[( \lambda _ { \mathrm { A } } + \lambda _ { \mathrm { B } } + \lambda _ { \mathrm { h } } ) \left(\left(\lambda_{\mathrm{A}}+\lambda_{\mathrm{B}}+\lambda_{\mathrm{h}}\right)\left(2 \lambda_{\mathrm{A}}+\lambda_{\mathrm{B}}+2 \lambda_{\mathrm{h}}\right)\right.\right.} \\
& \left.+\mu_{\mathrm{A}}\left(\lambda_{\mathrm{A}}+2 \lambda_{\mathrm{h}}\right)+\mu_{\mathrm{h}}\left(\left(2 \lambda_{\mathrm{A}}+\lambda_{\mathrm{B}}\right)\left(\lambda_{\mathrm{A}}+\lambda_{\mathrm{B}}+\lambda_{\mathrm{h}}\right)+\lambda_{\mathrm{B}} \mu_{\mathrm{A}}\right)\right]=0
\end{aligned}
$$

Mean time to system failure: The steady-state reliability of the system is given by:

$$
\begin{aligned}
R(s) & =P_{0}(s)+P_{1}(s)+P_{2}(s) \\
& =\frac{\left(s+x_{2}\right)\left(s+x_{3}\right)+2 \lambda_{A}\left(s+x_{3}\right)+2 \lambda_{h}\left(s+x_{2}\right)}{\prod_{r=1}^{3}\left(s-s_{r}\right)}
\end{aligned}
$$

The mean time to failure of the system is given by:

$$
\begin{aligned}
\text { MTTF }= & \lim _{s \rightarrow 0} R(s)=2 \lambda_{h}\left(\lambda_{A}+\lambda_{h}+\lambda_{B}+\mu_{A}\right) \\
& +\left(3 \lambda_{A}+\lambda_{h}+\lambda_{B}+\mu_{A}\right)\left(\lambda_{A}+\lambda_{h}+\lambda_{B}+\mu_{h}\right)^{\prime} \\
& \left(\left(2 \lambda_{A}+2 \lambda_{h}+\lambda_{B}\right)\left(\lambda_{A}+\lambda_{h}+\lambda_{B}+\mu_{A}\right)\right. \\
& \left.-2 \lambda_{A} \mu_{A}\right)\left(\lambda_{A}+\lambda_{h}+\lambda_{B}+\mu_{h}\right)-2 \lambda_{h} \mu_{h}\left(\lambda_{A}+\lambda_{h}+\lambda_{B}+\mu_{A}\right)
\end{aligned}
$$

System availability: The system availability is the probability that the system operates within the tolerances at a given instant of time and is obtained as follows: Using the infinitesimal generator shown in Fig. 1, we obtain the following differential equations:

$$
\begin{aligned}
& \frac{d p_{0}(t)}{d t}+\left(2 \lambda_{A}+2 \lambda_{h}+\lambda_{B}\right) p_{0}(t)=\mu_{A} p_{1}(t)+\mu_{h} p_{2}(t)+\mu_{B} p_{3}(t) \\
& \frac{d p_{1}(t)}{d t}+\left(\lambda_{A}+\lambda_{h}+\lambda_{B}+\mu_{A}\right) p_{1}(t)=2 \lambda_{A} p_{0}(t)+2 \mu_{A} p_{5}(t)+\mu_{h} p_{6}(t) \\
& \frac{d p_{2}(t)}{d t}+\left(\lambda_{A}+\lambda_{h}+\lambda_{B}+\mu_{h}\right) p_{2}(t)=2 \lambda_{h} p_{0}(t)+\mu_{A} p_{6}(t)+2 \mu_{h} p_{8}(t) \\
& \frac{d p_{3}(t)}{d t}+\mu_{B} p_{3}(t)=\lambda_{B} p_{0}(t)+\mu_{A} p_{4}(t)+\mu_{h} p_{0}(t) \\
& \frac{d p_{4}(t)}{d t}+\mu_{A} p_{4}(t)=\lambda_{B} p_{1}(t)
\end{aligned}
$$

$\frac{d p_{5}(t)}{d t}+2 \mu_{A} p_{5}(t)=\lambda_{A} p_{1}(t)$

$\frac{\mathrm{dp}_{6}(\mathrm{t})}{\mathrm{dt}}+\left(\mu_{\mathrm{A}}+\mu_{\mathrm{h}}\right) \mathrm{p}_{6}(\mathrm{t})=\lambda_{\mathrm{h}} \mathrm{p}_{1}(\mathrm{t})+\lambda_{\mathrm{A}} \mathrm{p}_{2}(\mathrm{t})$

$\frac{\mathrm{dp}_{7}(\mathrm{t})}{\mathrm{dt}}+\mu_{\mathrm{h}} \mathrm{p}_{7}(\mathrm{t})=\lambda_{\mathrm{B}} \mathrm{p}_{2}(\mathrm{t})$

$\frac{\mathrm{dp}_{8}(\mathrm{t})}{\mathrm{dt}}+2 \mu_{\mathrm{h}} \mathrm{p}_{8}(\mathrm{t})=\lambda_{\mathrm{h}} \mathrm{p}_{2}(\mathrm{t})$

Let $\mathrm{P}_{\mathrm{i}}(\mathrm{s})$ be the Laplace transform of $\mathrm{P}_{\mathrm{i}}(\mathrm{t}), \mathrm{i}=0,1$, $2, \ldots, 8$. Taking Laplace transform on both the sides of the differential Eq. 7-15 and using the initial $\sum_{i=0}^{8} P_{i}(t)=1$ conditions, solving for $\mathrm{P}_{\mathrm{i}}(\mathrm{s}), \mathrm{i}=0,1,2, \ldots, 8$ and inverting, we get $P_{i}(t), i=0,1,2, \ldots, 8$. Since $S_{0}, S_{1}$ and $\mathrm{S}_{2}$ correspond to system up-states, the system availability is given by:

$$
\begin{aligned}
& \operatorname{AV}(t)=P_{0}(t)+P_{1}(t)+P_{2}(t) \\
& =\frac{\mu_{h} \mu_{A} \mu_{B}\left(A_{0}-B_{0}+C_{0}\right)}{\prod_{r=1}^{9} s_{r}}+ \\
& \sum_{i=1}^{9} \frac{\left(s_{i}+\mu_{h}\right)\left(s_{i}+\mu_{A}\right)\left(s_{i}+\mu_{B}\right)\left(A_{i}-B_{i}+C_{i}\right)}{\prod_{r=1, r \neq i}^{9} s_{i}\left(s_{i}-s_{r}\right)} e^{s_{i} t}
\end{aligned}
$$

Where:

$$
\begin{aligned}
\mathrm{A}_{\mathrm{i}}= & 2 \lambda\left(\mathrm{s}_{\mathrm{i}}+2 \mu_{\mathrm{h}}\right)\left(\left(\mathrm{s}_{\mathrm{i}}\left(\mathrm{s}_{\mathrm{i}}+\mathrm{x}_{2}\right)+2 \mu_{\mathrm{A}}\left(\mathrm{s}_{\mathrm{i}}+\mathrm{x}_{2}-\lambda_{\mathrm{A}}\right)\right)\left(\mathrm{s}_{\mathrm{i}}+\mu_{\mathrm{A}}+\mu_{\mathrm{h}}\right)\right. \\
& \left.+\left(\mathrm{s}_{\mathrm{i}}+\mu_{\mathrm{A}}\right)\left(\lambda_{\mathrm{A}} \mu_{\mathrm{A}}-2 \lambda_{\mathrm{h}} \mu_{\mathrm{h}}\right)\right) \\
\mathrm{B}_{\mathrm{i}}= & 2 \lambda_{\mathrm{A}}\left(\mathrm{s}_{\mathrm{i}}+2 \mu_{\mathrm{A}}\right)\left(2 \lambda_{\mathrm{h}} \mu_{\mathrm{h}}\left(\mathrm{s}_{\mathrm{i}}+\mathrm{x}_{4}\right)+\left(\mathrm{s}_{\mathrm{i}}+2 \mu_{\mathrm{h}}\right)\right. \\
& \left.\left(\lambda_{\mathrm{A}} \mu_{\mathrm{A}}-\lambda_{\mathrm{h}} \mu_{\mathrm{h}}-\left(\mathrm{s}_{\mathrm{i}}+\mathrm{x}_{4}\right)\left(\mathrm{s}_{\mathrm{i}}+\mathrm{x}_{3}\right)\right)\right)
\end{aligned}
$$




$$
\begin{aligned}
\mathrm{C}_{\mathrm{i}}= & -2 \lambda_{\mathrm{h}} \mu_{\mathrm{h}}\left(\left(\mathrm{s}_{\mathrm{i}}+\mathrm{x}_{4}\right)\left(\mathrm{s}_{\mathrm{i}}\left(\mathrm{s}_{\mathrm{i}}+\mathrm{x}_{2}-\lambda_{\mathrm{A}}\right) \mu_{\mathrm{A}}\right)-\lambda_{\mathrm{h}} \mu_{\mathrm{h}}\left(\mathrm{s}_{\mathrm{i}}+2 \mu_{\mathrm{A}}\right)\right) \\
& +\left(\mathrm{s}_{\mathrm{i}}+2 \mu_{\mathrm{h}}\right)\left(-2 \lambda_{\mathrm{A}} \mu_{\mathrm{A}}\left(\left(\mathrm{s}_{\mathrm{i}}+\mathrm{x}_{3}\right)\left(\mathrm{s}_{\mathrm{i}}+\mathrm{x}_{4}\right)-\lambda_{\mathrm{A}} \mu_{\mathrm{A}}\right)\right. \\
& +\left(\mathrm{s}_{\mathrm{i}}+2 \mu_{\mathrm{A}}\right)\left(-\lambda_{\mathrm{A}} \mu_{\mathrm{A}}\left(\mathrm{s}_{\mathrm{i}}+\mathrm{x}_{2}\right)\right. \\
& \left.\left.+\left(\mathrm{s}_{\mathrm{i}}+\mathrm{x}_{3}\right)\left(\left(\mathrm{s}_{\mathrm{i}}+\mathrm{x}_{2}\right)\left(\mathrm{s}_{\mathrm{i}}+\mathrm{x}_{4}\right)-\lambda_{\mathrm{h}} \mu_{\mathrm{h}}\right)\right)\right) \\
\mathrm{A}_{0}= & 4 \lambda_{\mathrm{h}} \mu_{\mathrm{h}} \mu_{\mathrm{A}}\left(\mu_{\mathrm{A}}\left(2 \mathrm{x}_{2}-\lambda_{\mathrm{A}}\right)+2 \mu_{\mathrm{h}}\left(\mathrm{x}_{2}-\lambda_{\mathrm{A}}-\lambda_{\mathrm{h}}\right)\right) \\
\mathrm{B}_{0}= & -8 \lambda_{\mathrm{A}} \mu_{\mathrm{A}} \mu_{\mathrm{h}}\left(\mathrm{x}_{4} \lambda_{\mathrm{B}}+\lambda_{\mathrm{A}}\left(\mathrm{x}_{4}-\mu_{\mathrm{A}}\right)+\mu_{\mathrm{h}}\left(\mathrm{x}_{4}+\lambda_{\mathrm{h}}\right)\right) \\
\mathrm{C}_{0}= & 4 \mu_{\mathrm{h}} \mu_{\mathrm{A}}\left(\left(\lambda_{\mathrm{A}}-\mathrm{x}_{2}\right)\left(\lambda_{\mathrm{A}} \mu_{\mathrm{A}}+\mathrm{x}_{4}\left(\lambda_{\mathrm{h}}-\mathrm{x}_{3}\right)\right)+\lambda_{\mathrm{h}} \mu_{\mathrm{h}}\left(\lambda_{\mathrm{h}}-\mathrm{x}_{3}\right)\right)
\end{aligned}
$$

where, $s_{i}$ the roots of the polynomial of the expand the determinately of the following matrix:

$$
\left[\begin{array}{ccccccccc}
\left(\mathrm{s}+\mathrm{x}_{1}\right) & -\mu_{\mathrm{A}} & -\mu_{\mathrm{h}} & -\mu_{\mathrm{B}} & 0 & 0 & 0 & 0 & 0 \\
-2 \lambda_{\mathrm{A}} & \left(\mathrm{s}+\mathrm{x}_{2}\right) & 0 & 0 & 0 & -2 \mu_{\mathrm{A}} & -\mu_{\mathrm{h}} & 0 & 0 \\
-2 \lambda_{\mathrm{h}} & 0 & \left(\mathrm{~s}+\mathrm{x}_{3}\right) & 0 & 0 & 0 & -\mu_{\mathrm{A}} & 0 & -2 \mu_{\mathrm{h}} \\
-\lambda_{\mathrm{B}} & 0 & 0 & \left(\mathrm{~s}+\mu_{\mathrm{B}}\right) & -\mu_{\mathrm{A}} & 0 & 0 & -\mu_{\mathrm{h}} & 0 \\
0 & -\lambda_{\mathrm{B}} & 0 & 0 & \left(\mathrm{~s}+\mu_{\mathrm{A}}\right) & 0 & 0 & 0 & 0 \\
0 & -\lambda_{\mathrm{A}} & 0 & 0 & 0 & \left(\mathrm{~s}+2 \mu_{\mathrm{A}}\right) & 0 & 0 & 0 \\
0 & -\lambda_{\mathrm{h}} & -\lambda_{\mathrm{A}} & 0 & 0 & 0 & \left(\mathrm{~s}+\mathrm{x}_{4}\right) & 0 & 0 \\
0 & 0 & -\lambda_{\mathrm{B}} & 0 & 0 & 0 & 0 & \left(\mathrm{~s}+\mu_{\mathrm{h}}\right) & 0 \\
0 & 0 & -\lambda_{\mathrm{h}} & 0 & 0 & 0 & 0 & 0 & \left(\mathrm{~s}+2 \mu_{\mathrm{h}}\right)
\end{array}\right]
$$

Where:

$$
\begin{aligned}
& \mathrm{x}_{1}=\left(2 \lambda_{\mathrm{A}}+2 \lambda_{\mathrm{h}}+\lambda_{\mathrm{B}}\right) \\
& \mathrm{x}_{2}=\left(\lambda_{\mathrm{A}}+\lambda_{\mathrm{h}}+\lambda_{\mathrm{B}}+\mu_{\mathrm{A}}\right) \\
& \mathrm{x}_{3}=\left(\lambda_{\mathrm{A}}+\lambda_{\mathrm{h}}+\lambda_{\mathrm{B}}+\mu_{\mathrm{h}}\right) \\
& \mathrm{x}_{4}=\left(\mu_{\mathrm{A}}+\mu_{\mathrm{h}}\right)
\end{aligned}
$$

Steady-state availability: The steady-state availability of the system is given by:

$$
\begin{aligned}
\mathrm{AV}_{0}(\infty)= & \operatorname{lims}_{\mathrm{s} \rightarrow 0} \mathrm{sV}_{0}(\mathrm{~S}) \\
= & \mu_{\mathrm{h}} \mu_{\mathrm{A}} \mu_{\mathrm{B}}\left[4 \lambda _ { \mathrm { h } } \mu _ { \mathrm { h } } \mu _ { \mathrm { A } } \left(\mu_{\mathrm{A}}\left(2 \mathrm{x}_{2}-\lambda_{\mathrm{A}}\right)\right.\right. \\
& +2 \mu_{\mathrm{h}}\left(\mathrm{x}_{2}-\lambda_{\mathrm{A}}-\lambda_{\mathrm{h}}\right)-8 \lambda_{\mathrm{A}} \mu_{\mathrm{A}} \mu_{\mathrm{h}}\left(\mathrm{x}_{4} \lambda_{\mathrm{B}}+\lambda_{\mathrm{A}}\left(\mathrm{x}_{4}-\mu_{\mathrm{A}}\right)\right. \\
& \left.+\mu_{\mathrm{h}}\left(\mathrm{x}_{4}+\lambda_{\mathrm{h}}\right)\right)+4 \mu_{\mathrm{h}} \mu_{\mathrm{A}}\left(\left(\lambda_{\mathrm{A}}-\mathrm{x}_{2}\right)\left(\lambda_{\mathrm{A}} \mu_{\mathrm{A}}+\mathrm{x}_{4}\left(\lambda_{\mathrm{h}}-\mathrm{x}_{3}\right)\right)\right. \\
& \left.\left.+\lambda_{\mathrm{h}} \mu_{\mathrm{h}}\left(\lambda_{\mathrm{h}}-\mathrm{x}_{3}\right)\right)\right] / \\
& {\left[4 \mu _ { \mathrm { A } } \mu _ { \mathrm { h } } \left(\lambda_{\mathrm{A}}^{3} \mu_{\mathrm{B}} \mu_{\mathrm{h}}^{2}+\lambda_{\mathrm{A}}^{2} \mu_{\mathrm{h}}\left(\mu_{\mathrm{B}} \mu_{\mathrm{A}}\left(\lambda_{\mathrm{B}}+2 \lambda_{\mathrm{h}}\right)\right.\right.\right.} \\
& \left.+\mu_{\mathrm{h}}\left(2 \lambda_{\mathrm{B}} \mu_{\mathrm{A}}+\mu_{\mathrm{B}}\left(3 \lambda_{\mathrm{B}}+\lambda_{\mathrm{h}}+3 \mu_{\mathrm{A}}\right)\right)+\mu_{\mathrm{B}} \mu_{\mathrm{h}}^{2}\right) \\
& +\mu_{\mathrm{A}}\left(\lambda_{\mathrm{B}} \mathrm{x}_{4}+\mu_{\mathrm{A}}\left(\lambda_{\mathrm{h}}+\mathrm{x}_{4}\right)\right)\left(\lambda_{\mathrm{B}}^{2} \mu_{\mathrm{h}}+\mu_{\mathrm{B}}\left(\lambda_{\mathrm{h}}+\mu_{\mathrm{h}}\right)^{2}\right. \\
& \left.\left.+\lambda_{\mathrm{B}}\left(2 \lambda_{\mathrm{h}}+\mu_{\mathrm{h}}\right)\left(\mu_{\mathrm{B}}+\mu_{\mathrm{h}}\right)\right)+\lambda_{\mathrm{B}}\left(2 \lambda_{\mathrm{h}}+\mu_{\mathrm{h}}\right)\left(\mu_{\mathrm{B}}+\mu_{\mathrm{h}}\right)\right) \\
& +\lambda_{\mathrm{A}}\left(\lambda_{\mathrm{B}}^{2} \mu_{\mathrm{h}}\left(2 \mu_{\mathrm{A}}\left(\mu_{\mathrm{A}}+\mu_{\mathrm{B}}\right)+\mu_{\mathrm{h}}\left(3 \mu_{\mathrm{A}}+2 \mu_{\mathrm{B}}\right)\right)\right. \\
& +\mu_{\mathrm{B}} \mu_{\mathrm{A}}\left(\lambda_{\mathrm{h}}+\mu_{\mathrm{h}}\right)\left(\lambda_{\mathrm{h}}\left(\mu_{\mathrm{h}}+\mathrm{x}_{4}\right)+\mu_{\mathrm{h}}\left(3 \mu_{\mathrm{A}}+2 \mu_{\mathrm{h}}\right)\right) \\
& +\lambda_{\mathrm{B}}\left(2 \lambda_{\mathrm{h}} \mathrm{x}_{4}\left(\mu_{\mathrm{A}} \mu_{\mathrm{B}}+\mu_{\mathrm{h}}\left(\mu_{\mathrm{A}}+\mu_{\mathrm{B}}\right)\right)\right. \\
& \left.\left.+\mu_{\mathrm{h}}\left(2 \mu_{\mathrm{A}}^{2} \mu_{\mathrm{B}}+\mu_{\mathrm{A}} \mu_{\mathrm{h}}\left(3 \mu_{\mathrm{A}}+5 \mu_{\mathrm{B}}\right)+2 \mu_{\mathrm{h}}^{2}\left(\mu_{\mathrm{A}}+\mu_{\mathrm{B}}\right)\right)\right)\right)
\end{aligned}
$$

Special case: When repair failure is not allowed i.e., Let $9 \mu_{\mathrm{k}}, \mu_{\mathrm{A}}, \mu_{\mathrm{k}}=0$ ).

Reliability analyses without repair failure: Using the infinitesimal generator given in Fig. 1 when repair failure is not allowed, we derive the following differential equations:

$$
\begin{aligned}
& \frac{\mathrm{dp}_{0}(\mathrm{t})}{\mathrm{dt}}+\left(2 \lambda_{\mathrm{A}}+2 \lambda_{\mathrm{h}}+\lambda_{\mathrm{B}}\right) \mathrm{p}_{0}(\mathrm{t})=0, \\
& \frac{\mathrm{dp}_{1}(\mathrm{t})}{\mathrm{dt}}+\left(\lambda_{\mathrm{A}}+\lambda_{\mathrm{h}}+\lambda_{\mathrm{B}}\right) \mathrm{p}_{1}(\mathrm{t})=2 \lambda_{\mathrm{A}} \mathrm{p}_{0}(\mathrm{t}) \\
& \frac{\mathrm{dp}_{2}(\mathrm{t})}{\mathrm{dt}}+\left(\lambda_{\mathrm{A}}+\lambda_{\mathrm{h}}+\lambda_{\mathrm{B}}\right) \mathrm{p}_{2}(\mathrm{t})=2 \lambda_{\mathrm{h}} \mathrm{p}_{0}(\mathrm{t})
\end{aligned}
$$

Let $\mathrm{P}_{\mathrm{i}}(\mathrm{s})$ be the Laplace transform of $\mathrm{P}_{\mathrm{i}}(\mathrm{t}), \mathrm{i}=0,1$, 2. Taking Laplace transform on both the sides of the differential equations given above, solving for $\mathrm{P}_{\mathrm{i}}(\mathrm{t})$, $i=0,1,2$ and inverting, we get $P_{i}(t), i=0,1,2$. Then the system reliability is given by:

$$
\begin{aligned}
R(t) & =P_{0}(t)+P_{1}(t)+P_{2}(t) R(t) \\
& =2 e^{-\left(\lambda_{A}+\lambda_{h}+\lambda_{B}\right) t}-e^{-\left(2 \lambda_{A}+2 \lambda_{h}+\lambda_{B}\right) t}
\end{aligned}
$$

Mean time to system failure without repair failure: Then the steady-state reliability of the system without repair failure is given by:

$$
\begin{aligned}
\mathrm{R}(\mathrm{s}) & =\mathrm{P}_{0}(\mathrm{~s})+\mathrm{P}_{1}(\mathrm{~s})+\mathrm{P}_{2}(\mathrm{~s}) \\
& =\frac{\left(\mathrm{s}+3 \lambda_{\mathrm{A}}+3 \lambda_{\mathrm{h}}+\lambda_{\mathrm{B}}\right)}{\left(\mathrm{s}+\lambda_{\mathrm{A}}+\lambda_{\mathrm{h}}+\lambda_{\mathrm{B}}\right)\left(\mathrm{s}+2 \lambda_{\mathrm{A}}+2 \lambda_{\mathrm{h}}+\lambda_{\mathrm{B}}\right)}
\end{aligned}
$$

The mean time to failure of the system without repair failure is given by:

$$
\operatorname{MTTF}=\frac{\left(3 \lambda_{A}+3 \lambda_{h}+\lambda_{B}\right)}{\left(2 \lambda_{A}+2 \lambda_{h}+\lambda_{B}\right)\left(\lambda_{A}+\lambda_{h}+\lambda_{B}\right)}
$$

Setting $\lambda_{\mathrm{A}}=0.004, \lambda_{\mathrm{B}}=0.005 \lambda_{\mathrm{h}}=0.003, \mu_{\mathrm{A}}=0.2$, $\mu_{\mathrm{B}}=0.2$ and $\mu_{\mathrm{h}}=0.1$ in Eq. 5 and 20 we get Table 1.

\begin{tabular}{lcll}
\multicolumn{4}{l}{ Table 1: Reliability of the two systems at any time } \\
\hline Sr. No. & $\mathrm{t}$ & $\mathrm{R}(\mathrm{t})$ for first system & $\mathrm{R}(\mathrm{t})$ for second system \\
\hline 1 & 0 & 1.00000 & 1.00000 \\
2 & 10 & 0.94944 & 0.94688 \\
3 & 20 & 0.89766 & 0.88939 \\
4 & 30 & 0.84870 & 0.82983 \\
5 & 40 & 0.80239 & 0.76990 \\
6 & 50 & 0.75864 & 0.71088 \\
7 & 60 & 0.71727 & 0.65369 \\
8 & 70 & 0.67816 & 0.59894 \\
9 & 80 & 0.64117 & 0.54707 \\
10 & 90 & 0.60620 & 0.49833 \\
\hline
\end{tabular}


Table 2: Variation of MTTF with respect to $\lambda_{\mathrm{A}}$ of the two systems

\begin{tabular}{llcc}
\hline Sr. No. & $\lambda_{\mathrm{A}}$ & MTTF for first system & MTTF for second system \\
\hline 1 & 0.001 & 190.7390 & 145.2991 \\
2 & 0.002 & 187.1698 & 133.3333 \\
3 & 0.003 & 183.2433 & 122.9947 \\
4 & 0.004 & 179.0294 & 114.0351 \\
5 & 0.005 & 174.5940 & 106.2271 \\
6 & 0.010 & 151.0039 & 78.8530 \\
7 & 0.020 & 108.8940 & 51.8207 \\
8 & 0.030 & 9.8163 & 38.5471 \\
9 & 0.040 & 60.7727 & 30.6777 \\
10 & 0.050 & 48.0263 & 25.4737 \\
\hline
\end{tabular}

Table 3: Variation of MTTF with respect to $\lambda_{\mathrm{B}}$ of the two systems

\begin{tabular}{llcc}
\hline Sr. No. & $\lambda_{B}$ & MTTF for first system & MTTF for second system \\
\hline 1 & 0.001 & 624.3900 & 183.3330 \\
2 & 0.002 & 384.9850 & 159.7220 \\
3 & 0.003 & 278.2800 & 141.1760 \\
4 & 0.004 & 217.8860 & 126.2630 \\
5 & 0.005 & 179.0290 & 114.0350 \\
6 & 0.010 & 94.6338 & 75.9804 \\
7 & 0.020 & 48.7011 & 44.6623 \\
8 & 0.030 & 32.7828 & 31.3268 \\
9 & 0.040 & 24.7051 & 24.0347 \\
10 & 0.050 & 19.8201 & 19.4627 \\
\hline
\end{tabular}

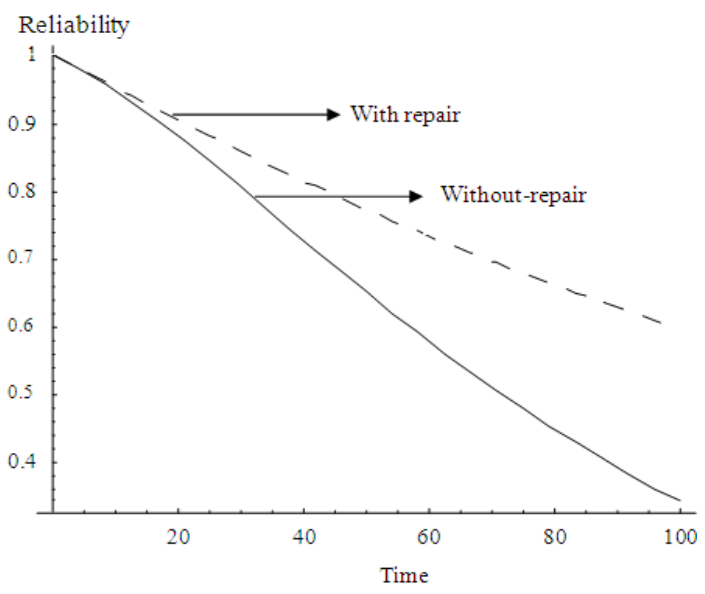

Fig. 3: Reprecent the relation between reliability and time

Setting $\lambda_{\mathrm{B}}=0.005, \lambda_{\mathrm{h}}=0.003, \mu_{\mathrm{A}}=0.2, \mu_{\mathrm{B}}=0.2$ and $\mu_{\mathrm{h}}=0.1$ in Eq. 7 and 22 we get Table 2 .

Setting $\lambda_{\mathrm{A}}=0.004, \lambda_{\mathrm{h}}=0.003, \mu_{\mathrm{A}}=0.2, \mu_{\mathrm{B}}=0.2$ and $\mu_{\mathrm{h}}=0.1$ in Eq. 6 and 21 we get Table 3 .

interpretation of the results: To observe the effect of the repair on the system behavior, we plot the represent Mean Time To Failure (MTTF) and the reliability at any time t, against $\lambda_{\mathrm{A}}$ (hardware failure rate of a unit for sub-system A), $\lambda_{h}$ (human failure rate of a unit for sub-system A) and $\lambda_{B}$ (failure rate of a unit for subsystem B).

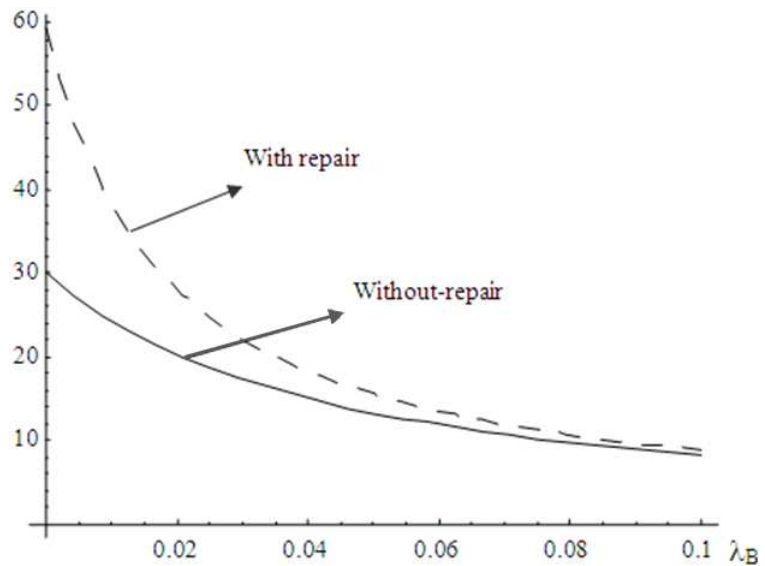

Fig. 4: Reprecent the relation between MTTF and $\lambda_{B}$

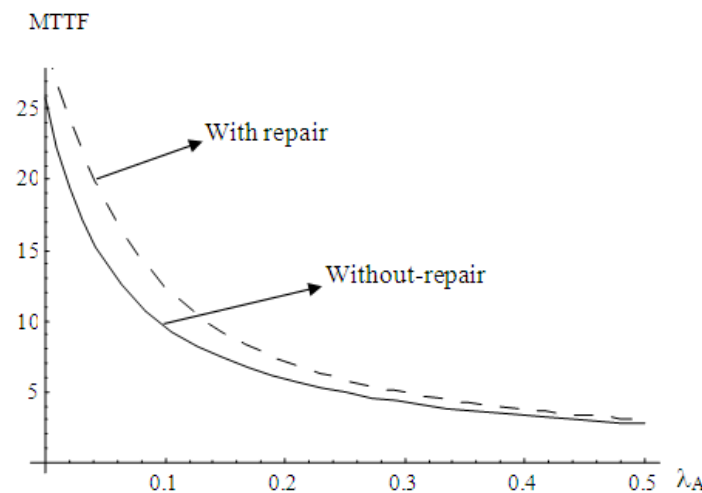

Fig. 5: Reprecent the relation between MTTF and $\lambda_{\mathrm{A}}$

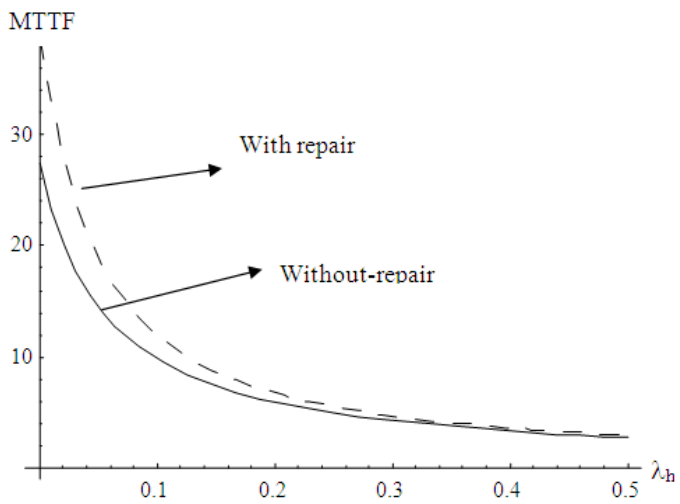

Fig. 6: Reprecent the relation between MTTF and $\lambda_{\mathrm{h}}$

Table 1-3 and Fig. 3-8 show that the present of an additional repair leads to improve the values of the Mean Time To Failure (MTTF) and the reliability at any time $t$ as shown from their behaviors when plotted against $\mathrm{t}, \lambda_{\mathrm{h}}, \lambda_{\mathrm{A}}$ or $\lambda_{\mathrm{B}}$. 


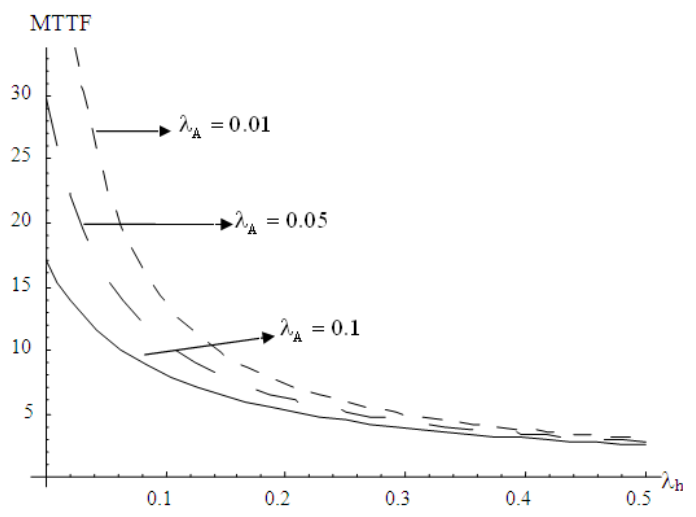

Fig. 7: Reprecent the relation between MTTF and $\lambda_{\mathrm{h}}$ with various values for $\lambda_{\mathrm{A}}$

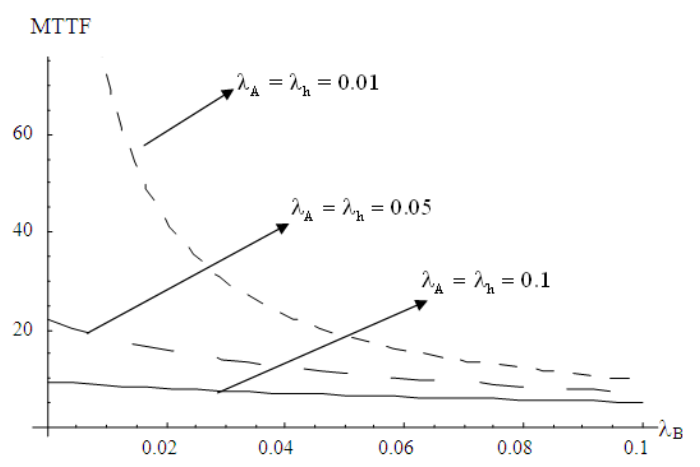

Fig. 8: Reprecent the relation between MTTF and $\lambda_{B}$ with various values for $\lambda_{\mathrm{A}}$ and $\lambda_{\mathrm{h}}$

\section{CONCLUSION}

Markov-renewal processes and properties of Laplace transforms has been used to measure the system effectiveness. The additional repair led to improve the values of the MTTF and the reliability at any time $\mathrm{t}$.

\section{REFERENCES}

1. Dillon, B.S., 1980. On human reliability bibliography. Microelect. Reliab., 20: 371-373.

2. Dillon B.S. and S.N. Rayayoti, 1985. Reliability analysis of non-maintained parallel systems subject to hardware failure and human error. Microelect. Reliab., 25: 111-122. http://cat.inist.fr/?aModele=afficheN\&cpsidt=9241702

3. Gupta, P.P. and Arvind Kumar, 1986. Reliability and MTTF analysis of non-repairable parallel redundant complex system under hardware and human failures. Microelect. Reliab., 26: 229-234. http://cat.inist.fr/?aModele $=$ afficheN\&cpsidt=8794399

4. Ritu Gupta, S.K. Mittal and C.M. Batra, 2006. Stochastic analysis of a compound redundant system involving human failure. J. Math. Stat., 2: 407-413.

http://www.journaldatabase.org/articles/141278/St ochastic_Analysis_of_a_.html 